\title{
Monetarisierung in der ökologischen Ökonomie!?
}

\section{Folgt aus dem Ansatz der ökologischen Ökonomie eine Abkehr von der monetären Bewertung von Natur und Landschaft? Eine nähere Betrachtung der damit gewonnenen Informationen legt nahe, daß auch eine ökologische Öko- nomie nicht auf diesen Bewertungsansatz verzichten kann. Aber sie kann wis- senschaftlich begründefe Grenzen für die Monetarisierung aufzeigen.}

S Von Jürgen Meyerboff toßen schon umweltökonomische Instrumente wie zum Beispiel Zertifikate vielfach auf Unverständnis und Ablehnung, so gilt dies für die monetäre Bewertung von Natur und Umwelt noch viel stärker. Ist sie doch selbst unter (Umwelt-) Ökonomen umstritten. Mit dem Aufkommen der ökologischen Ökonomie dürfte daher vielfach die Hoffnung verbunden gewesen sein, daß sie sich durch eine andere voranalytische Visison von der traditionellen Umweltökonomie abgrenzt, daß heißt, stärker den ökologischen Grundlagen des Wirtschaftens zuwendet und Konzepte wie die Monetarisierung aus ihrem Repertoire streicht. Doch allein ein Blick in die Hauszeitschrift „Ecological Economics“ zeigt sehr schnell und deutlich, daß die Monetarisierung darin einen recht breiten Raum einnimmt: Die Themenpalette reicht von der Analyse der Zahlungsbereitschaft für den Erhalt wildlebender Tierarten in Afrika bis hin zur Diskussion monetärer Indikatoren für Nachhaltigkeit. Eine Verfehlung der ökologischen Ökonomie oder doch hilfreicher und angebrachter Ansatz?

\section{- Die Analyse interdependenter Systeme}

In Absetzung von der neoklassischen Ökonomie geht die ökologische Ökonomie davon aus, daß das ökonomische System nicht unabhängig von den ökologischen Systemen analysiert werden kann. Ressourcennutzungen durch das ökonomische System können zu Veränderungen im ökologischen System führen, die aufgrund von Rückkoppelungseffekten wiederum entscheidend für die weitere Entwicklung der beiden miteinander verknüpften Systeme sind. Werden bestimmte Schwellenwerte erreicht, dann muß sogar von der Möglichkeit nicht-linearen
Systemverhaltens ausgegangen werden. Umwelt kann somit nicht als ein ,Sack voller Ressourcen" verstanden werden, aus dem die Ressourcen unabhängig voneinander entnommen werden können.

Diese wechselseitigen Beeinflussungen und die daraus resultierende Abhängigkeit des ökonomischen vom ökologischen System wurden bisher auch von der Ökonomie nicht angemessen berücksichtigt. Angestrebt wird daher eine ökologisch-ökonomische Interdependenzanalyse, in die die ökologische Umgebung als nutzungsabhängiges, veränderliches ökologisches System einbezogen wird. Die ökologische Ökonomie kann - vereinfacht - wie folgt charakterisiert werden (Perrings et al. 1995: 8):

- Ökonomisches und ökologisches System werden als gemeinsam determinierte Systeme verstanden. Darüber hinaus ist auch das Ausmaß der ökonomischen Aktivitäten (Scale) von Bedeutung. Es besteht die Möglichkeit von Rückkoppelungseffekten, die sowohl auf die Wohlfahrt derzeitiger als auch zukünftiger Generationen entscheidende Auswirkungen haben können.

- Die Dynamik der gemeinsam determinierten Systeme ist charakterisiert durch nicht-kontinuierliche Veränderungen im Bereich kritischer Schwellenwerte sowohl für biotische als auch für abiotische Ressourcen. Werden diese Schwellenwerte überschritten, dann reagiert das System in nicht mehr vorhersagbarer Weise. Die ökologische Ökonomie hat daher die Evolution nicht-linear ökologisch-ökonomischer Systeme zum Gegenstand, in denen Pfadabhängigkeit bedeutet, daß die bisherige Entwicklung bedeutend ist für heutige und zukünftige Entwicklungsmöglichkeiten.

- Die Stabilität des gesamten Systems hängt weniger von der Stabilität einzelner Ressourcen
- z. B. einzelner Tier- oder Pflanzenarten - als vielmehr von der Resilienz ab, also der Fähigkeit eines Systems, seine Selbstorganisation angesichts externer Störungen zu erhalten.

\section{Bewerfung in der ökologischen Ökonomie}

Kann die Analyse interdependenter Systeme noch als konsensfähig angesehen werden, so gehen die Schulen im Falle der Bewertung im Rahmen der ökologischen Ökonomie sehr deutlich auseinander: Auf der einen Seite finden sich Ökonomen, die im Anschluß an Arbeiten vor allem Krutillas (1967) mit seiner Unterscheidung in use- und non-use-values an der Weiterentwicklung und Anwendung der monetären Bewertung gearbeitet haben. Stellvertretend $\mathrm{zu}$ nennen sind D. Pearce, K. Turner, C. Perrings, E. B. Barbier, für Deutschland stellvertretend U. Hampicke. Auf der anderen Seite wurde von vielen Vertretern der ökologischen Ökonomie die monetäre Bewertung als ein völlig falscher Ansatz angesehen, da sie Märkte und die ihnen zugrundeliegenden Annahmen zum Ausgangspunkt der Bewertung machten, anstatt aus den Defiziten der Institution Markt die entsprechenden Lehren zu ziehen und andere Kriterien für die Bewertung heranzuziehen. Stellvertretend zu nennen sind P. Söderbaum, G. Munda, S. Funtowicz, M. Wackernagel, W. Rees, für Deutschland stellvertretend $\mathrm{F}$. Hinterberger et al.

$\mathrm{Zu}$ unterscheiden ist zunächst zwischen der Bewertung einzelner Projekte wie z. B. staatlicher Infrastrukturprojekte und der Entwicklung monetärer, gesamtgesellschaftlicher Indikatoren für Nachhaltigkeit. Vor allem der von Pearce und Atkinson (1993) vorgestellte monetäre Indikator für schwache Nachhaltigkeit hat sehr kontroverse Diskussionen ausgelöst. Hatte er doch u. a. zum Ergebnis, daß Länder wie Japan und die USA als ,schwach nachhaltig“ eingestuft wurden. Doch dieser Strang der Diskussion soll hier nicht weiter verfolgt werden. Vielmehr soll nach der Bedeutung der Monetarisierung für die Bewertung einzelner Projekte im Rahmen der ökologischen Ökonomie gefragt werden.

Eine zentrale Frage für die Bewertung generell ist zunächst, was gemessen und wessen Bewertung zur Entscheidungsfindung herangezogen werden soll. Nur wenn diese Fragen beantwortet sind, läßt sich eine Entscheidung über die geeignete Bewertungsmethode treffen: Denn mit Hilfe der Monetarisierung lassen sich ,nur“ Präferenzen für bestimmte Umweltzustände erfassen. 
Gefragt wird danach, welche Güterausstattung die Individuen wählen würden, wenn Umweltgüter auf Märkten nachgefragt werden könnten. Werden andere Informationen als diese gewünscht, dann sind auch andere Bewertungsmethoden heranzuziehen. Die Monetarisierung wird keine Informationen darüber liefern können, ob z. B. Infrastrukturprojekte wie Straßen mit dem Kriterium der ökologischen Nachhaltigkeit vereinbar sind.

Doch können umgekehrt Bewertungsmethoden wie Ecological Footprints (Wackernagel, Rees 1997) oder auch MIPS (Hinterberger et al.) wenn ihre schon in der Methode festgelegten Bewertungsgrundlagen denn akzeptiert werden - lediglich Handlungsfolgen verdeutlichen oder aufzeigen, was für eine nachhaltige Entwicklung notwendige Maßnahmen sind - wie z. B. die Reduktion der Stoffflüsse. Sie können aus sich selbst heraus aber keinerlei Legitimation für die Umsetzung ihrer Handlungsempfehlungen beanspruchen. Es sei denn, daß im Sinne einer Meritorisierung davon ausgegangen wird, Wissenschaftler und Politiker wüßten besser als andere, was richtig und gut für alle - vor allem spätere Generationen - ist.

Löst man sich bei der Monetarisierung von einer sehr engen ökonomischen Interpretation im Sinne des Anspruchs, das (pareto-)effiziente Bereitstellungsniveau öffentlicher Güter eindeutig ermitteln zu können, dann können die damit erfaßten individuellen Bewertungen im Sinne eines Referendums verstanden werden, das wichtige politische und ökonomische Informationen liefert (vgl. Zimmer 1994: 52). Auch können die Informationen als ein Maß für die individuellen Zielverfehlungskosten verstanden werden, die daraus resultieren, daß eine andere Quantität und Qualität öffentlicher Güter von der Politik bereitgestellt wird als von den Individuen gewünscht. Hohe Zielverfehlungskosten dürften aber auf Dauer zu Akzeptanzverlusten der Politik führen.

Folgt man dem Argument, daß sich mit Hilfe der Monetarisierung ökonomisch als auch politisch wichtige Informationen über Qualität und Quantität der vom Staat bereitzustellenden öffentlichen (Umwelt-)Güter gewonnen werden können, erscheint kein Grund ersichtlich, warum eine ökologische Ökonomie auf diese Art von Informationen verzichten sollte bzw. könnte. Es sei denn, wie schon gesagt, nicht mehr die betroffenen Individuen werden als maßgebend für die Güterbereitstellung bzw. für die umweltpolitische Zielfindung angesehen. Aus Sicht der ökologischen Ökonomie erscheint daher vielmehr eine Begrenzung der Monetarisierung wesentlich, die direkt an das Anliegen der ökologisch-ökonomischen Interdependenzanalyse anknüpft: Aufgrund von fundamentaler Unsicherheit über die Art der wechselseitigen Abhängigkeit und die Rolle einzelner Ressourcen für die Selbstorganisationsfähigkeit der ökologischen Systeme sind Grenzen für die Monetarisierung notwendig.

\section{Primäre Werte und Safe Minimum Standard}

Im Rahmen einiger Untersuchungen zum ökonomischen Wert von Feuchtgebieten haben Gren et al. (1994) die Unterscheidung zwischen primären und sekundären Werten getroffen.
Ausgangspunkt dafür war die Erkenntnis, daß nicht alle Werte, die ökologische Systeme für die menschliche Gesellschaft haben, über individuelle Präferenzen ermittelt werden können. Umgekehrt hängen aber diejenigen Werte, die sich mit Hilfe der individuellen Präferenzen bestimmen lassen, von der Existenz und Dauerhaftigkeit der Ökosysteme unmittelbar ab. Der gesamte soziale Wert von Ökosystemen kann danach wie folgt differenziert werden:

a) Produktion für die eigene Entwicklung und Erhaltung,

b) Export zu anderen Ökosystemen,

c) Export zur menschlichen Gesellschaft.

Als primäre Werte werden die unter a) genannten bezeichnet. Erfaßt werden damit diejenigen Bestandteile und Leistungen eines Ökosystems, die für die Selbstorganisationsfähigkeit des Systems und seine Aufrechterhaltung Voraussetzung sind. In bezug auf diese Funktionen sind sie nicht substituierbar, sondern komplementär. Individuelle Wertschätzung für eine Tier- oder Pflanzenart und ihre funktionale Bedeutung innerhalb des Ökosystem können aber auseinanderfallen, so daß die alleinige Orientierung an individuellen Präferenzen nicht hinreichend ist. Denn nur wenn die primären Werte gegeben sind, können auch die unter b) und c) genannten ökologischen Leistungen erbracht werden. Sie werden daher als sekundäre Werte bezeichnet. „But though such primary value is clearly a component of the economic value of environmental resources (it is an indirect use value) it is not directly measurable in terms of consumer preferences (...). That is, it is a public good whose role is subject

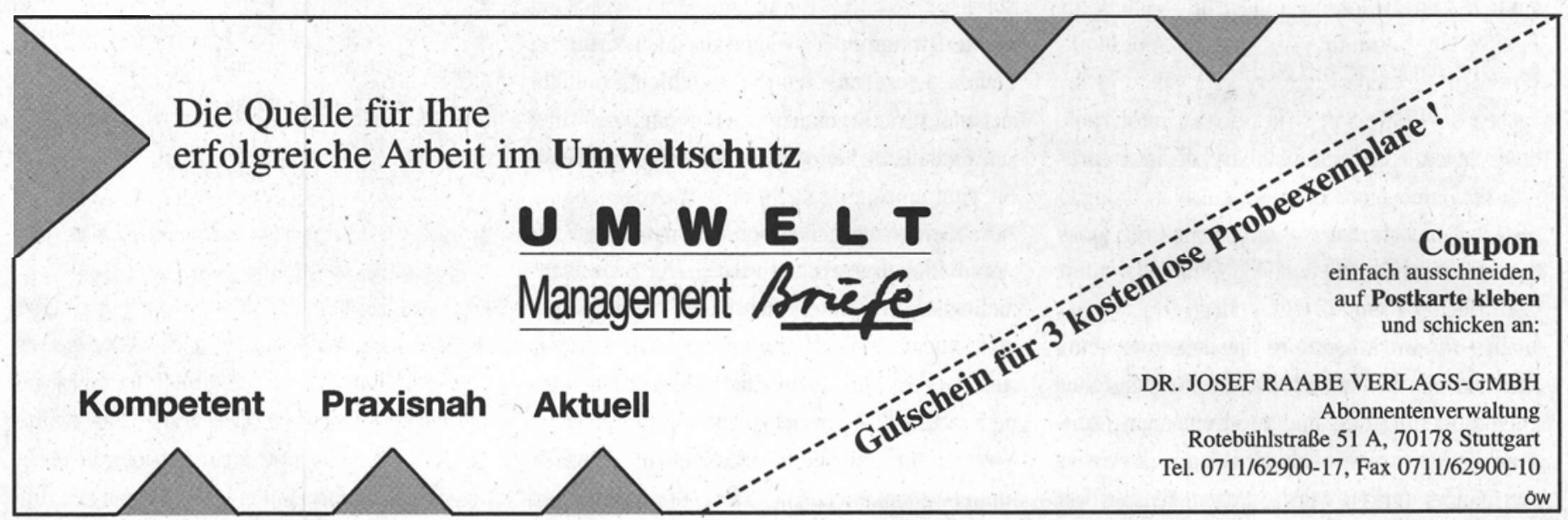


to fundamental uncertainty. Not only do economic agents seldom understand the role of functional ecosystems in the provision of goods and services, they also have a strong incentive to dissimulate about their preferences" (Perrings et al. 1995: 19).

Ein in der ökologischen Ökonomie zunehmend häufiger angeführtes Instrument in diesem Zusammenhang ist der von Ciriacy-Wantrup schon in den fünfziger Jahren in die Diskussion eingeführte Safe Minimum Standard (SMS). Mit ihm soll im Entscheidungsprozeß explizit die Unsicherheit und Irreversibilität möglicher Folgen der Nutzung natürlicher Ressourcen berücksichtigt werden. Grundidee dabei ist, daß Grenzen für - normale, alltägliche - ökonomische Abwägungen gesetzt werden, solange die Kosten dafür nicht inakzeptabel hoch sind. Als Kosten für die Anwendung des SMS werden die Nettonutzen der angestrebten Projektrealisierung angesehen.

Somit kann der SMS als ein Instrument zum Schutz der primären Werte verstanden werden: Mit ihm werden Grenzen für die alltägliche Abwägung zwischen Vor- und Nachteilen, also Kosten-Nutzen-Überlegungen, errichtet, die zu unabsehbaren Folgen führen könnten. Entscheidungen dieser Art, die z. B. dann gegeben sein könnten, wenn eine Tier- oder Pflanzenart als Folge der Projektrealisierung - auch lediglich regional - aussterben würde, sind aus dem üblichen routinemäßigen Entscheidungsablauf herauszunehmen und bedürfen gesonderter, kollektiv abgesicherter Entscheidunsgverfahren. „The SMS rule places biodiversity beyond the reach of routine trade-offs, where to give up ninety cents worth of biodiversity to gain a dollar worth of ground beef is to make a net gain. It also avoids claiming trump status for biodiversity, permitting some sacrifice of biodiversity in the face of intolerable costs to justify relaxation of the SMS. The idea of intolerable costs invokes an extraordinary decision process that takes biodiversity seriously by trying to distinguish costs that are intolerable from those that are merely substantial" (Randall, zitiert nach Bishop 1993: 72).

Doch Grundvoraussetzung für die Anwendung des SMS ist, daß ein Projekt bei Berücksichtigung aller negativen und positiven Auswirkungen überhaupt wirtschaftlich ist, d.h. zu einem Nettonutzen führen würde. Nur in diesem Fall sind Grenzen wie Safe Minimum Standards erforderlich.

\section{Ein Schritt vor dem anderen}

Gutachten und Stellungnahmen zu Kosten-Nutzen-Analysen im Rahmen der Bundesverkehrswegeplanung, die am IÖW erstellt wurden, haben gezeigt, daß viele Verkehrsprojekte entgegen den Ergebnissen der Gutachter im Auftrag des Bundesverkehrsministeriums zu keinem Nettonutzen führen würden, wenn auch die Auswirkungen auf Natur und Landschaft monetär bewertet würden. Trotz vieler berechtigt angeführter Mängel der Monetarisierung läßt sich daher die These wagen, daß es nicht nur im Naturschutz heute eine bessere Ausstattung mit öffentliche Gütern gäbe, wenn eine Monetarisierung in diesem Sinn konsequenter durchgefuihrt worden wäre. Verwiesen sei hier auf eine KostenNutzen-Analyse aus Österreich, an der sich dieses gut veranschaulichen läßt (vgl. Schönbäck et al. 1997): Überprüft werden sollte darin u.a., ob der Bau eines Flußkraftwerkes in der Donau oder die Maximalvariante eines Nationalparks Donau-Auen aus volkswirtschaftlicher Sicht die vorzuziehende Alternative sei. Berücksichtigt wurden alle relevanten Effekte wie Auswirkungen auf die Schiffahrt, den Grundwasserhaushalt, die Vorteile aufgrund kohlensioxid-freier Stromerzeugung durch das jeweilige Flußkraftwerk und auch die in einer Contingent Valuation ermittelte Wertschätzung für die Donau-Auen. Ergebnis war, daß der Verzicht auf die Flußkraftwerke und eine nur moderate Verbesserung der Schiffahrtsverhältnisse bei gleichzeitiger Umsetzung der Maximalvariante des Nationalparks die wirtschaftlichste Variante ist.

Es geht aber nicht um eine Huldigung der monetären Bewertung. Sie hat Grenzen, auf die insbesondere die ökologische Ökonomie aufmerksam macht. Aus ihren Erkennt- nissen heraus errichtete Grenzen für die Monetarisierung wie die primären Werte und der Safe Minimum Standard verdeutlichen dies. Auch geht es nicht darum, daß Ergebnisse der Monetarisierung politische Entscheidungen determinieren sollen. Sie sind einzig und allein als entscheidungsvorbereitend anzusehen. Doch bevor das Kind mit dem Bade ausgeschüttet wird, sollte auch angesichts des Ziels einer nachhaltigen Entwicklung sorgfältiger geprüft werden, welche Informationen welchen Einfluß auf politische Entscheidungen haben können. Der Nachweis, daß eine Vielzahl von Investitionsprojekten mit erheblichen Auswirkungen auf Natur und Landschaft entgegen offizieller Verlautbarung unwirtschaftlich sind, wäre in vielen Fällen schon ein großer
Fortschritt. Es könnte sich von daher empfehlen, zunächst erst einmal den Schritt der ökonomischen Bewertung von Projektfolgen auch auf Natur und Landschaft zu machen, bevor dieser Ansatz als von vornherein ungeeignet abqualifiziert wird. Zumal andere Bewertungsverfahen letztlich mit zumindest ähnlichen Problemen konfrontiert sind: So müssen Informationen oft auch zu einer einzigen Meßzahl - verdichtet und Gewichtungen festgelegt werden. Und andere Bewertungsverfahren garantieren auch nicht per se, daß eine ungleiche Verteilung der Zahlungsfähigkeit bei den Individuen „gerecht“ berücksichtigt wird etc.

In Österreich hat die besagte Untersuchung letztlich dazu geführt, daß auf den Bau der Kraftwerke verzichtet wurde. Nun kann sicher nicht davon ausgegangen werden, daß sich politische Entscheidungen tatsächlich an den Ergebnissen ökologisch bewußter Kosten-Nutzen-Analysen orientieren werden - aber dies ist ein Problem, unter dem nicht nur die monetäre Bewertung leidet.

\section{Literatur}

- Bishop, R.C. (1993): Economic Effiziency, Sustainability and Biodiversity. In: AMBIO, Heft 2-3, 69-73

- Gren, I.-M./Folke, C.,/Turner, K./Batemen, I. (1994): Primary and Secondary Values of Wetland Ecosystems. In: Journal of Environmental and Resource Economics

- Hinterberger, F./ Luks, F./Schmidt-Bleek, F. (im Erscheinen): Material flows vs. "natural capital". What makes an economy sustainable? In: Ecological Economics - Krutilla, J. V. (1967): Conservation Reconsidered. In: American Economic Review 57, 777 - 786

- Pearce, D. W./Atkinson, G. D. (1993): Copital theory and the measurement of sustainable development: an indicator of "weak" sustainability. In: Ecological Economics, 8, $103 \cdot 108$

- Perrings, C./Turner, R. K. \& Folke, C. (1995): Ecological Economics: The Study of Interdependent Economic and Ecological Systems. Discussionpaper des Beijer International Institute of Ecological Economics, Stockholm

- Schönbäck, W./Kosz, M./Madreiter, T. (1997): Nationalpark Donauaven: Kosten-Nutzen-Analyse. Wien/New York

- Zimmer, Y. (1994): Naturschutz und Landschaftspflege - Allokationsmechanismen, Pröferenzanalyse, Entwicklungspotentiale. Kiel

- Wackernagel, M./Rees, W. E. (1997): Perceptual and Structural Barriers to Investing in Natural Capital. Economics from an Ecological Footprint Perspective. Volume $20 / 1$ 
(c) 20I0 Authors; licensee IÖW and oekom verlag. This is an article distributed under the terms of the Creative Commons Attribution Non-Commercial No Derivates License (http://creativecommons.org/licenses/by-nc-nd/3.o/), which permits unrestricted use, distribution, and reproduction in any medium, provided the original work is properly cited. 